\title{
Policy and guidance
}

\section{Ensuring Prudent Use Of Antimicrobials In Human Medicine In The EUROPEAN UnION, 2005}

\author{
G Werner ${ }^{1,3}$, S Bronzwaer ${ }^{1,2}$ \\ 1. European Commission, Public Health Directorate, Luxembourg \\ 2. European Food Safety Authority (EFSA), Parma, Italy \\ 3. Robert Koch-Institute, Wernigerode Branch, Germany
}

The overuse and misuse of antibiotics pose a serious danger to public health by contributing to the development of bacteria resistant to treatment. In 2001, the European Commission launched a strategy to combat the threat of antimicrobial resistance to human, animal and plant health, which includes data collection, surveillance, research, awareness-raising exercises and the phasing out of antibiotics for non-medical use in animals. The Council Recommendation on the prudent use of antibiotics in human medicine adopted in 2002 was a component in this strategy, outlining clear-cut measures in human medicine that EU Member States could take to reduce antimicrobial resistance. This report summarises the main actions taken at Member State and Community level and highlights the areas of the Recommendation needing further attention. The report outlines a variety of measures already taken by Member States in line with the Recommendation, including improved surveillance of antibiotic use and resistance, and closer cooperation between different professionals on this issue. Member States have taken good steps forward in putting measures in place against antimicrobial resistance. However, some key areas need to be better addressed, in particular infection control, reducing self-medication with antibiotics and educating health professionals and the general public on the proper use of antimicrobial treatments. The report remarks that self-medication with antibiotics is still a problem in many Member States: a 'prescription-only' approach should be strictly enforced and educational activities are needed.

\section{Introduction}

Overuse and misuse of antibiotics have contributed to the development and spread of bacteria that are resistant to treatment, therefore posing a serious danger to public health [1-5]. In 2001, the European Commission (hereafter referred to as the Commission) launched a strategy [6] to combat the threat of antimicrobial resistance to human, animal and plant health, which includes data collection, surveillance, research, awareness-raising exercises and the phasing out of antibiotics for non-medical use in animals. The Council Recommendation on the prudent use of antibiotics in human medicine [7] (hereafter referred to as Recommendation) is an important component in this strategy, as described earlier in this journal [8]. The Recommendation asks Member States and EEA (European Economic Area) countries to put in place specific strategies on prudent use of antimicrobial agents. These strategies should include measures for the surveillance of antimicrobial resistance and use, control and preventive measures, education and training, and research, and the countries should report to the Commission on its implementation.
Methods

The Commission convened representatives from several Member States in a working group on the prudent use of antimicrobial agents in human medicine that developed a template for reporting. This template was designed in the form of a questionnaire to facilitate reporting in a concise and comparable manner, allowing for better collation and analysis of the information. Member States and EEA countries were asked to report to the Commission on the implementation of the Recommendation. Templates were sent to all Permanent Representations that coordinated the response through their national contact points and responsible institutions. During 2004, the Commission received one coordinated reply from every Member State, from Iceland and Norway, and also from the acceding country Bulgaria, which responded voluntarily. Results were summarised by the Commission and recorded with the help of the working group in a report to the Council highlighting the areas of the Recommendation needing further attention. [9] This report is supported by a Commission staff working paper providing a more detailed analysis, as well as tables summarising answers from single Member States. [10] For more details the reader is referred to these Commission papers available on the Commission's website; in this paper, the authors limit themselves to presenting the main findings and recommendations.

\section{Results}

\section{National strategies and intersectoral mechanism}

Countries specified whether they have a national strategy to contain the problem of antimicrobial resistance and whether a national action plan has been formulated. To coordinate the implementation of strategies and exchange information with the Commission and other Member States, the Council of the EU recommended that each Member State should have in place rapidly and possibly by November 2002 an appropriate intersectoral mechanism (IM).

Sixteen Member States reported to have a national strategy and 10 countries are developing such a strategy to address the problem of antimicrobial resistance. Nine countries have formulated a national action plan and in 14 countries it is under preparation. Twenty Member States, two EEA countries and Bulgaria have an $I M$ in place and five countries are creating one. The old Member States and the EEA countries indicated that the IM in their country was set up prior to the Recommendation (between 1995 and 2001) meaning that already established committees or structures have taken the form of IM. There are major differences in responsibilities, objectives, and composition of the IM in the Member States. Also there is considerable variation in the legal status of the IM between 
countries. In some countries there is specific legislation governing the IM, giving it executive powers, while in other countries the IM is merely an advisory body.

\section{Surveillance systems for antimicrobial resistance}

The Recommendation asked Member States to establish or strengthen their surveillance systems on antimicrobial resistance in order to gather reliable and comparable data on the susceptibility of pathogens.

The European Antimicrobial Resistance Surveillance System (EARSS) is a network of national surveillance systems, currently comprising about 800 laboratories from 31 countries that facilitate the collection of European data on antimicrobial resistance in indicator bacteria from invasive infections (Streptococcus pneumonioe, Stophylococcus oureus, Enterococcus foecium/foecolis, Escherichio coli) in a common format. EARSS extended surveillance as from 2005 also to Pseudomonos oeruginoso and Klebsiello pneumonioe. Also other surveillance networks monitor susceptibility of pathogens: Enter-net (International surveillance network for enteric infections) performs surveillance of human Solmonello, Compylobocter ond Verocytotoxin-producing E. coli 0157 infections, and EuroTB (Surveillance of tuberculosis in Europe) performs surveillance of tuberculosis (including (multi-)drug resistant TB). Susceptibility of meningococci, gonococci and Treponemo pollidum (syphilis) is also being monitored through EU-wide surveillance networks.

In 18 countries the IM is coordinating activities on antimicrobial resistance surveillance, in 13 countries the IM is actively collecting data, and in 19 countries the IM is proposing actions based on the findings. Fourteen countries reported having ownership of antimicrobial resistance data clearly defined. However, several obstacles for surveillance were reported: primarily the lack of a sustained financial basis, unclear legal status and regulation of privacy issues. Sixteen countries have published a national report on antimicrobial resistance. In nine Member States, two EEA countries and Bulgaria, formal collaboration between human and veterinary medicine has been established. In all but one of the new Member States, such interaction is lacking. Data are mostly collected from routine laboratory systems and in most countries distinction can be made between hospital-acquired and community-acquired strains. All but two countries report routinely having external quality assurance systems in place to verify validity of data.

\section{Surveillance systems for antimicrobial use}

Member States are requested to establish or strengthen a surveillance system for the collection of data on prescription and use of antimicrobial agents at the appropriate levels to allow monitoring of overall use involving, among others, prescribers, pharmacists and other parties collecting such data. Since the adoption of the Recommendation, the EU-funded project ESAC (European Surveillance of Antimicrobial Consumption) has been established. All Member States, EEA countries and Bulgaria participate in the ESAC project, which has facilitated the collection of European data on antimicrobial use in a common format.

All countries have some activities in place, but these do not always have country-wide coverage. In most countries other bodies than the IM are coordinating activities on antimicrobial consumption surveillance. In about half of the countries financial or legal obstacles are encountered in obtaining surveillance data. Linkage of antibiotic consumption data with clinical indications is available in few countries, mostly as specific research projects. Likewise, no single country can link consumption with resistance on a continuous basis. Only six countries have developed indicators to monitor prescribing practices of antimicrobial agents. The majority of Member States coordinate actions to improve prescribing practices, although the scope and target groups vary. Although many countries provide some kind of feedback to prescribers, no country provides continuous feedback on the prescribing practices of prescribers. In six Member States and two EEA countries collaboration with veterinary surveillance is established, but in none of the new Member States such a formal link is reported.

\section{Control and preventive measures}

Member States are requested to implement control and preventive measures to support the prudent use of antimicrobial agents and to limit the spread of communicable diseases.

Seven countries reported that antimicrobials sold without a prescription are still considered to be a relevant source of inappropriate antimicrobial use. No country was able to estimate the current proportion of all antimicrobials (systemic and local) sold without prescription. Therefore the Commission has cofunded a project on self-medication and antimicrobial resistance (SAR) to assess the problem of self-medication with antibiotics in Europe. Results show that antimicrobial drug self-medication is a cause for concern in Europe [11]. Sixteen countries have measures in place to enforce regulations for prescription-only use of systemic antimicrobials. Such measures are requested through the Community pharmaceutical legislation on medicinal products for human use [12].

Most countries have nationally accepted guidelines on appropriate use of antimicrobials for most common infections and medical interventions, such as surgical prophylaxis, otitis media, sinusitis, tonsillopharyngitis, community-acquired pneumonia, urinary tract infections and meningitis. The impact of these guidelines on prescribing practices is monitored in few countries and if so, it does only include single indications. In none of the new Member States prescribing practices are monitored.

Twenty countries have regulations about the pharmaceutical industry's sponsorship of, and gifts or other inducements to prescribers. Twenty two countries allow free antimicrobial samples to be given to the prescriber, and nine allow free samples of antibiotics in phase IV trials. Fifteen countries monitor whether regulations on sponsoring are respected and 14 have a disciplinary system in place to enforce these regulations. Sixteen countries have a control system on good practice of marketing of antimicrobial agents in place. Such measures are requested through the Community pharmaceutical legislation on medicinal products for human use. [12]

Twenty two countries have a national programme for hospital hygiene and infection control in place, and in 18 it is mandatory for each hospital to have an infection control committee. Only about half of the countries have legal requirements or recommendations about the number of infection control nurses per hospital bed, and have an accreditation procedure for hospitals and/or nursing homes. Only 14 countries require infection control to be a part of the hospital accreditation procedure, and even fewer require it to be part of nursing homes' accreditation procedure. Eighteen countries have national guidelines for the control of multiresistant pathogens, however, this often includes only a single pathogen (mostly methicillin-resistant $S$. aureus (MRSA)). 


\section{Education and training}

The Recommendation asks Member States to promote education and training of health professionals on the problem of antimicrobial resistance and to inform the general public about the importance of prudent use of antimicrobial agents.

The report shows that not all countries provide students entering the healthcare professions with education on the appropriate use of antimicrobials. However, continuing education on selected issues is provided later in their career. In all countries education is provided by non-sponsored continuing education, and almost all countries also provide such continuing education funded through sponsorship by the pharmaceutical industry. Eighteen countries have carried out reports and studies on the perception and knowledge of the public and health professionals on topics related to antimicrobial resistance. This most often concerns knowledge and perception on appropriate antimicrobial use. All but six countries have performed campaigns of some kind within the past five years to raise awareness on topics related to antimicrobial resistance. The campaigns usually addressed health professionals rather than the general public. A number of countries did not address the general public at all, or usually addressed them with messages concerning vaccination programmes only.

\section{Commission support for EU action}

The Commission has made antimicrobial resistance a priority in its public health and research programmes. It works closely with Member States through representatives from the IM in the working group on the prudent use of antimicrobial agents in human medicine and has funded a number of key activities contributing about $€ 2.8$ million over the past three years from the Public Health Programme, accounting for approximately $10 \%$ of the 'health threat' budget. Under the Framework Programmes for Research and Technological Development, a broad range of projects related to antimicrobial resistance is currently funded at an annual average EU contribution of approximately $€ 20$ million. The project portfolio encompasses research on basic mechanisms of emergence and transmission of resistance, development of new drugs and diagnostic tests as well as clinical and epidemiological research. For more details, see: http://www.cordis.lu/lifescihealth/major/drugs.htm.

\section{Conclusions and recommendations}

It should be noted that Member States provided information solely by self-assessment, and for this reason the authors have avoided any ranking or judgement system. Independent assessments would help by obtaining more objective data. The Commission welcomed the establishment of the European Centre for Disease Prevention and Control (ECDC) and believes that it will have an important role in operating European surveillance in the area of antimicrobial resistance. ECDC could engage in independent assessment visits, as have been carried out last year together by ECDC, the Commission and the World Health Organization to assess Member States' preparedness for influenza.

One of the requirements of effective implementation is a clear action plan and sufficient resources. Member States that have not yet put in place a national strategy nor formulated a national action plan are encouraged to do so. No specific recommendations have been made as to the nature of the $I M$ and indeed the status, organisational structure, funding, and understanding of the responsibilities of the IM between the Member States reveal major differences. To ensure implementation of national strategies, it is advisable to better define and possibly strengthen responsibilities, budget, and structure of the IM. All Member States should ensure that the IM is truly intersectoral and should consider extending IM membership to involve representatives from veterinary medicine.

Although EU surveillance networks have helped to make antimicrobial resistance data better comparable between countries, problems remain at local level (hospitals) where trend data are often not available to steer policies. Other recommendations on surveillance of antimicrobial resistance are to establish links between human and veterinary medicine in all countries and ensure that external quality assessment are done at regular intervals.

Significant progress has been made in surveillance of antimicrobial use. However, the coverage and possibilities of breakdown of data for antimicrobial use could be improved in many countries. Linkage of antimicrobial consumption data with indications for use is an essential tool to monitor compliance with treatment guidelines but is available only in a minority of countries. Also feedback to prescribers on their prescribing practices is still under development in many countries. As for surveillance of resistance also in surveillance of antimicrobial use structural collaboration with other sectors is lacking in many countries including all new Member States.

Systemic antibacterial medicinal products are still obtained without a prescription in a number of Member States and therefore all countries should have clear measures in place to enforce 'prescription-only' use of systemic antibiotics. Other sources of self-medication with antibiotics may include leftover drugs from treatment courses prescribed earlier or drugs obtained from relatives or friends, and the SAR study showed that this occurred in all countries studied [11]. This problem of self-medication should be tackled appropriately in all Member States in particular through education of the general public.

All countries should have nationally accepted guidelines in place recommending appropriate antibiotic treatment, at least for the most common human infections and interventions, and the impact of these guidelines on prescribing practices should be assessed regularly. A significant number of countries (in particular new Member States) has neither established regulations about sponsoring of and/or gifts or inducements to prescribers by the pharmaceutical industry, nor do they monitor whether regulations on sponsoring are respected. Regulations need to be in place according provisions of Community pharmaceutical legislation on medicinal products for human use.

Although in six countries relevant education on antimicrobial resistance for students entering the healthcare professions is missing, overall it seems that education of professionals is addressed. Further EU-wide exchange of best practice on education and campaigns addressing the public would be beneficial. This exchange of good practice could extend to an exchange on vaccination campaigns and hygiene/infection control.

Healthcare institutions are strongly recommended to step up infection control measures to counter the spread of so-called superbugs such as MRSA. Institutions should have their own infection control systems or committees in place or ensure that relevant tasks are undertaken by other appropriate existing bodies. Countries should consider making infection control part of an accreditation or other quality control procedure for hospitals and possibly nursing homes. The Commission has recently concluded 
a public consultation on this important subject and is working on an appropriate proposal in the area of infection control [13].

In conclusion, while it is true that most Member States have taken a variety of actions as requested by the Recommendation, efforts need to be stepped up to reduce the inappropriate use of antibiotics in the EU and curb the problem of resistance. The report of the Commission has been sent to the Council for their consideration and possible action to ensure a full and speedy implementation of the Council Recommendation in all Member States.

\section{Acknowledgements}

The authors wish to thank the members of the "Working group on the prudent use of antimicrobial agents in human medicine" for their valuable support.

\section{Note}

This article is a detailed analysis of measures taken by Member States and recommendations for action on the basis of Member States' reports on the implementation of the Council Recommandation (2002/77/EC) on the prudent use of antimicrobial agents in human medicine.

\section{References}

1. Smith DL, Harris AD, Johnson JA, Silbergeld EK, Morris JG Jr. 2002. Animal antibiotic use has an early but important impact on the emergence of antibiotic resistance in human commensal bacteria. Proc Natl Acad Sci U S A. 2002;99(9):6434-9.

2. Helmuth R, Hensel M. 2004. Towards the Rational Use of Antibiotics: Results of the First International Symposium on the Risk Analysis of Antibiotic Resistance. J Vet Med Ser. 2004;B 51:357-60.

3. Joint FAO/OIE/WHO Expert Workshop on Non-Human Antimicrobial Usage and Antimicrobial Resistance: Scientific assessment. Geneva, Dec 1-5, 2003. pp. 1-40.

4. Courvalin P. Antimicrobial drug resistance: "Prediction is very difficult, especially about the future”. Emerg Infect Dis. 2005;11(12):1503-1506.
5. Vander Stichele RH, Elseviers MM, Ferech M, Blot S, Goossens H. Hospital consumption of antibiotics in 15 European countries: results of the ESAC retrospective data collection (1997-2002). J Antimicrob Chemother. 2006;58(1):159-67.

6. Commission of the European Communities. Communication from the commission on a community strategy against antimicrobial resistance. Com (2001) 333 final 20.06.2001. Available from: http://europa.eu.int/eurlex/en/com/cnc/2001/act333en01/com2001_0333en01-01.pdf

7. European Council. Council Recommendation of 15.11.2001 on the Prudent Use of Antimicrobial Agents in human medicine (2002/77/EC). 0J L34 of 5.2.2002, p.13.

8. Bronzwaer S, Lonnroth A, Haigh R. The European Community Strategy Against Antimicrobial Resistance Euro Surveill. 2004 Jan;9(1):30-4. Available from: http://www.eurosurveillance.org/em/v09n01/0901-223.asp

9. Commission of the European Communities. Report from the Commission to the Council on the basis of Member States' reports on the implementation of the Council Recommendation (2002/77/EC) on the prudent use of antimicrobial agents in human medicine. COM (2005) 0684 final 22.12.2005. Available from: http://ec.europa.eu/health/ph_threats/com/mic_res/com684_en.pdf. Accessed 17 January 2007.

10. Commission of the European Communities. Commission Staff Working Document. Detailed analysis of Member States' reports on the implementation of the Council Recommendation (2002/77/EC) on the prudent use of antimicrobial agents in human medicine. SEC (2005) 1746 of 22.12.2005. Available from: http://ec.europa.eu/health/ph threats/com/mic res/am 051222_en.pdf. Accessed 17 January 2007.

11. Grigoryan L, Haaijer-Ruskamp FM, Burgerhof JGM, Mechtler R, Deschepper R, Tambic-Andrasevic A, et al. Self-medication with antimicrobial drugs in Europe. Emerg Infect Dis. 2006 Mar;12(3):452-9.

12. Commission of the European Communities. Directive 2001/83/EC of the European Parliament and of the Council of 6 November 2001 on the Community code relating to medicinal products for human use as last amended by Directive 2004/27/EC 0.J. L 136, 30/4/2004 p. 34 - 57. 0.J. L311 of 28.11.2001, p.67.

13. European Commission. Public consultation on strategies for improving patient safety by prevention and control of healthcare-associated infections. December 2005. Available from: http://europa.eu.int/comm/health/ph threats/ com/comm_diseases_cons01_en.htm. Accessed 17 January 2007.

'The informotion contoined in this publicotion does not necessorily reflect the opinion or the position of the Europeon Commission

Citation: Werner G, Bronzwaer S. Ensuring prudent use of antimicrobials in huma medicine in the European Union, 2005. Euro Surveill 2007;12(1)[Epub ahead of print]. Available online: http://www.eurosurveillance.org/em/v12n01/1201-225.asp 